\title{
SUBNOTIFICAÇÃO DE ACIDENTES BIOLÓGICOS PELA ENFERMAGEM DE UM HOSPITAL UNIVERSITÁRIO ${ }^{1}$
}

\author{
BIOLOGICAL ACCIDENTS UNDERREPORTING BY NURSING \\ PROFESSIONALS OF A UNIVERSITY HOSPITAL
}

\author{
EL SUBREGISTRO DE LOS ACCIDENTES BIOLÓGICOS POR EL \\ PERSONAL DE ENFERMERÍA DE UN HOSPITAL UNIVERSITARIO
}

\author{
Milene Dias Ferreira* \\ Flaviana Regina Pimenta ${ }^{* *}$ \\ LUIZA TAYAR FACCHIN ${ }^{* * *}$ \\ ELUCIR GIR ${ }^{* * * *}$ \\ Sílvia Rita Marin da Silva Canini ${ }^{* * * *}$
}

\begin{abstract}
RESUMO
Objetivo: Identificar a taxa de subnotificação de acidentes com material biológico pela equipe de enfermagem de um hospital universitário e os motivos referidos para a subnotificação. Material e método: Pesquisa de corte transversal. Foram entrevistados individualmente todos os profissionais de enfermagem que prestavam serviços assistenciais aos pacientes do hospital em estudo ou que manuseavam objetos utilizados por eles e que concordaram participar da pesquisa. Os dados foram analisados por meio de estatística descritiva. O estudo foi aprovado pelo Comitê de Ética e Pesquisa do hospital. Resultados: A taxa de subnotificação de acidentes foi 36,6\%. O motivo mais relatado para justificar a subnotificação foi considerar o acidente como de baixo risco (52,7\%). Conclusão: Acredita-se que o este estudo possa favorecer o conhecimento dos profissionais sobre seu risco ocupacional e contribuir para proposição de medidas que aumentem a adesão dos profissionais ao procedimento de notificação e assim conferir maior segurança à saúde desses trabalhadores.
\end{abstract}

Palavras chave: Notificação de acidente de trabalho, exposição a agentes biológicos, equipe de enfermagem.

\begin{abstract}
Objective: To identify the rate of underreporting of accidents with biological material by the nursing staff

${ }^{1}$ Artigo abstraído da dissertação de mestrado intitulada: Subnotificação de acidentes com material biológico pelos profissionais de enfermagem de um hospital-escola do interior paulista. Fonte de Apoio: CNPq.

* Enfermeira. Mestre em Ciências da Saúde, pelo Departamento de Enfermagem Geral e Especializada, Escola de Enfermagem de Ribeirão Preto da Universidade de São Paulo. Ribeirão Preto, Brasil. E-mail: milenediasferreira@yahoo.com.br

** Educadora física. Doutora em Ciências da Saúde pelo Programa Interunidades de Doutoramento em Enfermagem, Escola de Enfermagem de Ribeirão Preto da Universidade de São Paulo. Ribeirão Preto, Brasil. E-mail: prof.flaviana@ibrmrp.com. br

*** Enfermeira. Doutora em Ciências da Saúde pela Escola de Enfermagem de Ribeirão Preto da Universidade de São Paulo. Ribeirão Preto, Brasil. E-mail: luiza@eerp.usp.br

${ }^{x * x+t}$ Enfermeira. Professor Titular do Departamento de Enfermagem Geral e Especializada, Escola de Enfermagem de Ribeirão Preto da Universidade de São Paulo. Ribeirão Preto, Brasil. E-mail: egir@eerp.usp.br

${ }^{* * * * *}$ Enfermeira. Professor Associado do Departamento de Enfermagem Geral e Especializada, Escola de Enfermagem de Ribeirão Preto da Universidade de São Paulo. Ribeirão Preto, Brasil. E-mail: canini@eerp.usp.br
\end{abstract}


of a university hospital and the reasons cited for the underreporting. Method: Cross-sectional research. We individually interviewed all nursing professionals who provided health care services to patients at the study hospital or who manipulated objects used by them and who agreed to participate. Data were analyzed using descriptive statistics. The study was approved by the Research Ethics Committee of the hospital. Results: The rate of underreporting of accidents was $36.6 \%$. The most frequently reported reason to justify the underreporting was considered the accident as low risk (52.7\%). Conclusion: It is believed that the present study may promote the knowledge of professionals about their occupational risk and will contribute to propose measures to improve compliance professionals to the notification procedure and consequently give greater security to the health of these workers.

Key words: Occupational accidents registry, exposure to biological agents, nursing, team.

\section{RESUMEN}

Objetivo: Identificar el subregistro de los accidentes con material biológico por el personal de enfermería de un hospital universitario y las razones citadas para el subregistro. Material y método: Investigación transversal. Se entrevistó individualmente a todas las enfermeras que prestaron los servicios de atención médica a los pacientes en el hospital en estudio o manipularon objetos usados por ellos y que aceptaron participar. Los datos fueron analizados utilizando estadística descriptiva. El estudio fue aprobado por el Comité Ético de Investigación del hospital. Resultados: El subregistro de los accidentes fue de 36,6\%. La razón más frecuente para justificar el subregistro fue considerar el accidente como de bajo riesgo (52,7\%). Conclusión: Se cree que este estudio puede promover el conocimiento de los profesionales sobre su riesgo laboral y contribuya a proponer medidas para aumentar la adherencia de los profesionales para el procedimiento de notificación y por lo tanto dar una mayor seguridad para la salud de estos trabajadores.

Palabras clave: Notificación de accidentes del trabajo, exposición a agentes biológicos, grupo de enfermería.

Fecha recepción: 03/03/14 Fecha aceptación: 16/06/15

\section{INTRODUÇÃO}

A obtenção de dados sobre notificação de acidentes de trabalho envolvendo material biológico possibilita aos órgãos gestores das instituições relacionar todos os fatores implicados na ocorrência dos acidentes, verificar os motivos mais frequentes, buscar soluções baseadas nas informações contidas no registro, implementar ações corretivas e avaliar a eficácia das mesmas (1). Entretanto, estudos demonstram que muitos incidentes com material biológico são subnotificados $(2,3)$.

Os profissionais da área da saúde (PAS) se expõem à diferentes tipos de risco no ambiente de trabalho, os quais podem comprometer sua saúde física e/ou mental (4).
Entre esses riscos, o biológico pode expor os PAS a patógenos por meio de contato com sangue e/ou fluido contaminados, sendo que já foram descritos, na literatura, 60 espécies de patógenos transmitidos após exposição ocupacional. Os vírus de maior relevância epidemiológica, responsáveis pela maioria das infecções ocupacionais relatadas na literatura (5), são os vírus da hepatite B (VHB), vírus da hepatite $\mathrm{C}$ (VHC) e vírus da imunodeficiência humana (VIH).

Os PAS que atuam em hospitais estão mais expostos a estes patógenos pois a prevalência desses vírus é maior em pacientes internados que na população geral (6).

Os acidentes com material biológico atingem todas as categorias de PAS, porém, estudos apontam que os profissionais de en- 
fermagem são os que mais se acidentam e os que sustentam os maiores índices de soroconversão ao VHI $(7,8)$.

As exposições ocupacionais a material biológico potencialmente contaminado podem ocorrer por meio de picadas de agulhas, ferimentos com objetos cortantes (via percutânea), contato direto das mucosas oral, nasal ou ocular, pelo contato com a pele não íntegra e/ou por mordedura humana (9).

A conduta pós-exposição ocupacional a material biológico inclui dois aspectos: o atendimento especializado para que seja avaliada a gravidade do acidente e iniciadas medidas profiláticas específicas para minimizar os riscos de soroconversão ao VIH e ao VHB e a notificação do acidente por meio da Comunicação de Acidente de Trabalho (CAT), para garantir os aspectos da legislação trabalhista, e do preenchimento da ficha de notificação do Sistema de Informação de Agravos de Notificação (SINAN) (10).

Autores apontam que muitos profissionais acidentados não completam o seguimento clínico (11) ou não realiza nenhuma medida profilática pós exposição ocupacional (12) e que o número de acidentes pode ser bem maior devido a subnotificação (13).

A notificação da exposição a material biológico permite ações de vigilância em saúde do trabalhador, pois por meio dos dados sobre acidentes ocorridos é possível avaliar as causas mais frequentes e implementar medidas preventivas de acordo com a realidade de cada local (10).

Reconhecendo-se a importância da notificação de acidentes com material biológico, as altas taxas de subnotificação e os riscos a que os profissionais de enfermagem se expõem diariamente, julgou-se relevante realizar esta pesquisa cujos objetivos foram analisar a taxa de subnotificação de acidentes com material biológico ocorridos entre a equipe de enfermagem de um hospital escola nos últimos 12 meses, e os motivos referidos para que não preenchessem a Comunicação de Acidente de Trabalho.

\section{MATERIAL E MÉTODO}

Estudo de corte transversal, realizado por meio da busca ativa dos casos de subnotificação de acidentes com material biológico pela equipe de enfermagem de um hospital universitário.

Trata-se de um hospital de ensino, integrado ao Sistema Único de Saúde (SUS), que desenvolve atividades voltadas ao ensino, assistência e pesquisa científica. Atende pessoas do município de Ribeirão Preto, cidade localizada no interior do estado de São Paulo. É um centro de referência e, por isso, também atende casos mais complexos encaminhados de outras cidades ou estados. Possui 866 leitos de internação.

A população do estudo foi composta por todos os profissionais da equipe de enfermagem (enfermeiros, auxiliares e técnicos de enfermagem) que atuavam no referido hospital no período da coleta de dados e que aceitaram participar da pesquisa. Os critérios de inclusão foram: profissional de enfermagem atuante no hospital em estudo e que prestava serviços assistenciais diretos aos pacientes ou manuseava objetos utilizados por eles. Os critérios de exclusão foram: profissional de enfermagem que não prestava serviços assistenciais diretos aos pacientes ou não manuseava objetos utilizados por eles; profissionais que estavam afastados do trabalho; aqueles que se recusaram a participar do estudo e aqueles que foram entrevistados na fase de teste da pesquisa. Após a anuência do enfermeiro chefe, do diretor de cada setor e da Divisão de Enfermagem do hospital, os profissionais foram abordados no próprio local de trabalho e convidados a participar da pesquisa. Os profissionais que tinham disponibilidade de participação na pesquisa no momento em que foram abordados já foram entrevistados e os profissionais que estavam indisponíveis no momento foram procurados novamente em horário agendado por eles. As entrevistas foram realizadas individualmente em lo- 
cal privativo, dentro do setor de trabalho do profissional participante após assinatura do Termo de Consentimento Livre e Esclarecido. A coleta de dados foi realizada em todos os turnos de trabalho (manhã, tarde e noite) e em todos os dias da semana. A duração das entrevistas variou de 4 a $12 \mathrm{~min}$. Esta etapa da pesquisa procedeu-se no período de 24/04/2009 à 30/04/2011.

Quanto aos acidentes envolvendo material biológico, foram considerados apenas os que ocorreram no próprio hospital com os profissionais durante o seu turno de trabalho.

Para a coleta de dados foi elaborado um questionário contendo questões abertas e fechadas sobre os dados sociodemográficos, aspectos relacionados ao trabalho, ocorrência ou não de acidentes com material biológico, caracterização dos acidentes e conduta tomada após a exposição ocupacional com relação à notificação por meio da CAT e dados sobre os fatores relacionados à ausência de notificação.

$\mathrm{O}$ instrumento foi submetido à apreciação de três especialistas, que avaliaram a clareza e pertinência das questões, as sugestões foram acatadas e o instrumento foi considerado pertinente para o alcance dos objetivos propostos.

Com o intuito de verificar o entendimento das questões, foram realizadas dez entrevistas individuais com profissionais de enfermagem, sendo que nenhuma dificuldade em responder as questões do instrumento foi identificada. As entrevistas não foram incluídas no presente estudo e os sujeitos envolvidos foram excluídos da pesquisa.

Os dados foram armazenados em um banco de dados estruturado em um programa de computador específico e analisados por meio de estatística descritiva.

$\mathrm{O}$ projeto de pesquisa foi aprovado pelo Comitê de Ética em Pesquisa do referido hospital com o processo No 8907/2008, se- guindo-se a Resolução do Conselho Nacional de Saúde No 466 de 2012 sobre diretrizes e normas regulamentadoras para pesquisas em seres humanos do Ministério da Saúde (14).

\section{RESULTADOS}

A população do estudo foi composta por todos os profissionais da equipe de enfermagem que prestavam assistência direta aos pacientes e/ou que manuseavam objetos utilizados por eles que atuavam no referido hospital no período da coleta de dados e que aceitaram participar da pesquisa.

Foram considerados elegíveis para participar da pesquisa 1.867 profissionais de enfermagem. Dos 201 (10,8\%) indivíduos que não participaram, $79(4,2 \%)$ estavam afastados por licença saúde, $10(0,5 \%)$ foram excluídos por terem sido entrevistados na fase de testes e $112(6,0 \%)$ recusaram-se a participar do estudo. A população do estudo foi composta por 1.666 profissionais de enfermagem, dos quais $352(21,1 \%)$ relataram ter sofrido pelo menos um acidente ocupacional envolvendo material biológico, no último ano, no hospital em estudo. Destes, 129 relataram não ter notificado pelo menos um acidente, portanto a taxa de subnotificação de acidentes envolvendo material biológico foi de $36,6 \%$.

Os técnicos de enfermagem (50,0\%) foram os profissionais que relataram maior número de subnotificação de acidente com material biológico ocorrido nos últimos 12 meses que antecederam a entrevista, seguido pelos auxiliares de enfermagem $(36,6 \%)$.

Observou-se que a subnotificação foi maior pelos trabalhadores com mais de um emprego (39,4\%), e entre aqueles que relataram ter recebido treinamento sobre prevenção e condutas após acidente ocupacional com material biológico $(38,1 \%)$.

Em relação ao tempo de experiência pro- 
fissional na enfermagem, observou-se maior subnotificação entre os que tinham menos de cinco anos de experiência $(40,0 \%)$ e mais de vinte anos (39,6\%). Quanto ao tempo de experiência na instituição, aqueles que referiram mais de vinte anos foram os que mais subnotificaram $(39,3 \%)$.

A subnotificação foi maior entre profissionais com menor jornada de trabalho semanal $(38,6 \%)$ e que trabalhavam no noturno $(43,7 \%)$.
Em relação ao tipo de acidente, observouse que os acidentes percutâneos $(80,7 \%)$ foram mais notificados que os acidentes envolvendo exposição cutânea $(48,1 \%)$. O sangue foi o fluido corporal mais frequentemente envolvido no acidente, tanto nos notificados por meio da CAT $(85,7 \%)$ quanto nos não notificados. A Tabela 1 apresenta a caracterização dos acidentes segundo o tipo de exposição e o material biológico envolvido.

Tabela 1. Caracterização dos acidentes envolvendo material biológico $(n=352)$ notificados ou não, segundo o tipo de exposição e o tipo de fluido envolvido do Hospital das Clínicas da Faculdade de Medicina de Ribeirão Preto. Ribeirão Preto, 2009-2011.

\begin{tabular}{|c|c|c|c|c|c|c|}
\hline \multirow[b]{3}{*}{ Variáveis } & \multicolumn{6}{|c|}{ Comunicação do acidente de trabalho } \\
\hline & \multicolumn{2}{|c|}{$\operatorname{Sim}(n=223)$} & \multicolumn{2}{|c|}{ Não $(n=129)$} & \multicolumn{2}{|c|}{ Total $(n=352)$} \\
\hline & $\mathbf{f}$ & $\%$ & $\mathrm{f}$ & $\%$ & f & $\%$ \\
\hline \multicolumn{7}{|l|}{ Tipo de Exposição } \\
\hline Percutânea & 180 & 80,7 & 43 & 33,3 & 223 & 63,3 \\
\hline Cutâneo-mucosa & 35 & 15,7 & 24 & 18,6 & 59 & 16,8 \\
\hline Cutâneo & 8 & 3,6 & 62 & 48,1 & 70 & 19,9 \\
\hline \multicolumn{7}{|l|}{ Fluido envolvido } \\
\hline Sangue & 191 & 85,7 & 103 & 79,8 & 294 & 85,5 \\
\hline Outro fluido sem sangue visível & 7 & 3,1 & 4 & 3,1 & 11 & 3,1 \\
\hline Outro fluido com sangue visível & 25 & 11,2 & 22 & 17,1 & 47 & 13,4 \\
\hline
\end{tabular}

Muitos foram os motivos relatados pelos profissionais acidentados como justificativa para a subnotificação. Contudo, o motivo mais frequentemente referido para a subnotificação foi a crença de que o acidente ocorrido apresentava baixo risco (52,7\%). $\mathrm{Na}$ categoria "baixo risco" foram agrupadas as respostas "o acidente não apresentou risco", "não foi percutâneo", "o paciente-fonte era criança", "o paciente-fonte era idoso" e "o paciente-fonte não tinha risco de doenças infectocontagiosas".

Outras razões também foram citadas como justificativa para a subnotificação da exposição ocupacional a material biológico. A Tabela 2 descreve cada desses motivos referidos segundo o tipo de exposição. 
Tabela 2. Distribuição dos motivos referidos pelos profissionais de enfermagem para a não realização da notificação segundo o tipo de exposição do Hospital das Clínicas da Faculdade de Medicina de Ribeirão Preto. Ribeirão Preto, 2009-2011.

\begin{tabular}{|c|c|c|c|c|c|c|c|c|}
\hline \multirow[b]{4}{*}{ Variáveis } & \multicolumn{6}{|c|}{ Tipo de exposição } & & \\
\hline & \multirow{2}{*}{\multicolumn{2}{|c|}{$\frac{\text { Percutânea }}{\mathrm{n}=43}$}} & \multirow{2}{*}{\multicolumn{2}{|c|}{$\begin{array}{c}\text { Cutâneo-mucosa } \\
\mathrm{n}=24 \\
\end{array}$}} & \multirow{2}{*}{\multicolumn{2}{|c|}{$\begin{array}{c}\text { Cutânea } \\
\mathrm{n}=62\end{array}$}} & \multirow{2}{*}{\multicolumn{2}{|c|}{$\begin{array}{c}\text { Total } \\
\mathrm{n}=129\end{array}$}} \\
\hline & & & & & & & & \\
\hline & $\mathrm{f}$ & $\%$ & $\mathrm{f}$ & $\%$ & $\mathrm{f}$ & $\%$ & $\mathrm{f}$ & $\%$ \\
\hline Baixo risco & 10 & 23,2 & 11 & 45,8 & 47 & 75,1 & 68 & 52,7 \\
\hline Burocracia & 8 & 18,6 & 1 & 4,1 & 0 & 0 & 9 & 7,0 \\
\hline Não considerou necessário & 2 & 4,6 & 1 & 4,1 & 6 & 9,7 & 9 & 7,0 \\
\hline Sorologia negativa do paciente & 5 & 11,6 & 1 & 4,1 & 2 & 3,2 & 8 & 6,2 \\
\hline Muito ocupado & 4 & 9,3 & 1 & 4,1 & 2 & 3,2 & 7 & 5,4 \\
\hline Ter que ficar até mais tarde & 5 & 11,6 & 1 & 4,1 & 0 & 0 & 6 & 4,7 \\
\hline Demora do procedimento & 2 & 4,6 & 2 & 8,3 & 1 & 1,6 & 5 & 3,9 \\
\hline Frequente e baixo risco & 0 & 0 & 3 & 12,5 & 1 & 1,6 & 4 & 3,1 \\
\hline Receio do chefe & 2 & 4,6 & 0 & 0 & 1 & 1,6 & 3 & 2,3 \\
\hline Orientação de colega ou supervisor & 1 & 2,3 & 1 & 4,1 & 1 & 1,6 & 3 & 2,3 \\
\hline Esquecimento & 1 & 2,3 & 1 & 4,1 & 0 & 0 & 2 & 1,6 \\
\hline Desconhecimento & 1 & 2,3 & 0 & 0 & 0 & 0 & 1 & 0,8 \\
\hline $\begin{array}{l}\text { Demora do procedimento e orientação } \\
\text { de outro profissional }\end{array}$ & 1 & 2,3 & 0 & 0 & 0 & 0 & 1 & 0,8 \\
\hline Agulha não utilizada no paciente & 1 & 2,3 & 0 & 0 & 0 & 0 & 1 & 0,8 \\
\hline Acidente ocorrido no final de semana & 0 & 0 & 1 & 4,1 & 0 & 0 & 1 & 0,8 \\
\hline Não lembra & 0 & 0 & 0 & 0 & 1 & 1,6 & 1 & 0,8 \\
\hline
\end{tabular}

\section{DISCUSSÃO E CONCLUSÃO}

O presente estudo investigou a taxa de subnotificação de acidentes com material biológico pela equipe de enfermagem de um hospital universitário do interior paulista e os motivos relatados para não notificarem legalmente os acidentes. A taxa de subnotificação encontrada foi de $36,6 \%$. Outros autores evidenciaram taxas de $64 \%$ (15), 80,3\% (16) e 79\% (17).

No Paquistão, autores revelaram que quase todos os acidentes ocorridos (99\%) não foram relatados (18). E na Suíça pesquisadores encontraram que apenas $41,4 \%$ das expo- sições à material biológico foram oficialmente notificados (19).

No presente trabalho, a taxa de subnotificação foi menor que as taxas encontradas em outros estudos, porém cabe ressaltar que a pesquisa foi desenvolvida em um hospitalescola, que tem investido em treinamento e possui um fluxograma específico para profissionais expostos à material biológico, fatores que podem favorecer a notificação de acidentes.

As categorias de técnicos e auxiliares de enfermagem foram as que mais subnotificaram os acidentes. Estudo corrobora este resultado uma vez que estes profissionais estão mais expostos devido às particularidades de 
suas atividades laborais (20).

Neste estudo, a variável treinamento parece não ter influenciado na notificação ou não dos acidentes ocorridos, pois a maioria dos acidentes subnotificados ocorreu com profissionais que relataram ter recebido pelo menos um treinamento na instituição. Pesquisa também revelou este resultado ao evidenciar que $84,1 \%$ dos acidentados foram previamente treinados (21).

Mais os acidentes mais subnotificados foram os cutâneos $(48,1 \%)$. Somando-se os acidentes cutâneos aos cutâneo-mucosos o porcentual foi ainda maior $(66,7 \%)$. Autores avaliaram a ocorrência e a subnotificação de acidentes ocorridos entre profissionais da saúde, e encontraram que os acidentes que mais frequentemente deixaram de ser notificados foram os cutâneo-mucosos (22).

Diversos estudos, em vários países, analisaram os motivos relatados como dificultadores para a notificação e encontraram, assim como no presente estudo, que a atribuição de baixo risco ao acidente foi fator motivador da subnotificação (22-24).

$\mathrm{Na}$ presente pesquisa $6,2 \%$ referiram que não notificaram por considerar que este era um procedimento desnecessário. Semelhante a este motivo referido, autores encontraram a "crença de que a notificação não traz nenhum benefício" como fator alegado para a subnotificação (25).

A burocracia foi citada como fator motivador da subnotificação em $7 \%$ dos acidentes ocorridos. Já revisão integrativa da literatura encontrou a burocracia como o motivo mais citado entre os artigos revisados (23). Estudo realizado entre médicos cirurgiões de diversas especialidades evidenciou que as razões mais citadas para a subnotificação foram a falta de tempo e o excesso de burocracia (26). "Estar muito ocupado" também foi evidenciado, nesta pesquisa, como motivo para a não realização da notificação. Outros autores também encontraram esta resposta e ainda a resposta "falta de tempo" $(23,27,28)$.

Esta pesquisa identificou que 3,9\% dos acidentados afirmaram não ter notificado o ocorrido devido à demora no procedimento de notificação. Pesquisa realizada nos Estados Unidos apontou que $42 \%$ dos profissionais acidentados não os notificaram devido ao procedimento consumir muito tempo. Os autores deste trabalho encontraram também que muitos profissionais subnotificaram seus acidentes por considerar que a notificação é um procedimento inútil (29).

O desconhecimento de como realizar a notificação também foi citado pelos neste trabalho. Diversas pesquisas evidenciaram este como um motivo alegado por profissionais como justificativa para a subnotificação $(22,23)$.

Medo da chefia foi referido por 2,3\% dos acidentados que subnotificaram a exposição biológica. Estudo realizado em um hospital de Chicago, entre os profissionais da área da saúde, corrobora com este resultado (22). Destaca-se que pesquisa realizada com enfermeiros de cinco hospitais escola no Teerã encontrou como fator mais importante para a subnotificação a insatisfação com investigações do funcionário após este ter notificado um acidente (30).

Estudo evidenciou que a taxa de subnotificação pode variar de acordo com o tipo de exposição, da categoria profissional estudada e do local onde o estudo foi conduzido, assim a subnotificação de acidentes com material biológico permanece como uma problemática que merece maior investimento (23).

Conclui-se que a notificação é um procedimento necessário para que as instituições de saúde possam avaliar os acidentes ocorridos e suas causas e assim elaborar, implementar e posteriormente avaliar estratégias preventivas, educacionais e corretivas. Estas medidas não se restringem apenas aos profissionais como também à própria instituição que pode perceber falhas que favoreçam a ocorrência de acidentes.

Contudo, observou-se $36,6 \%$ de subnotificação. Diferentes motivos foram alegados pela equipe de enfermagem como justifica- 
tiva para a não realizar o procedimento, merecendo destaque a subestimação do risco e por considerar o procedimento desnecessário, evidenciando a necessidade de conscientização destes profissionais.

Acredita-se que apesar de a taxa de subnotificação do presente estudo ser menor que a encontrada por outros pesquisadores, os motivos alegados pelos profissionais podem subsidiar ações concretas para aumentar a adesão dos profissionais ao procedimento de notificação e assim conferir maior segurança a sua saúde.

\section{REFERÊNCIAS}

1. Ministério da Saúde (BR). Secretaria de Atenção à Saúde, Departamento de Ações Programáticas Estratégicas. Exposição a material biológico [Internet]. Brasília, DF: Ministério da Saúde; 2006 [citado 4 dezembro 2013]. 76 p. Disponível em: http://bvsms.saude.gov.br/bvs/publicacoes/protocolo_expos_mat_biologicos. pdf

2. Mendonça KM, Tipple AFV, Sousa ACS, Pereira MS, Rapparini C. Acidentes com material biológico em serviços de urgência e emergência. Cienc. enferm. 2014: 20(2): 65-71.

3. Alves AP, Ferreira MD, Prearo MP, Gir E, Canini SRMS. Subnotificação de acidentes ocupacionais com material biológico pela enfermagem no bloco cirúrgico. Rev. Eletr. Enf. [Internet]. 2013 [citado 10 jul 2015]; 15(2): 375-81. Disponível em: http://h200137217135.ufg.br/index.php/ fen/article/view/18554

4. Dalri RCMB, Robazzi MLCC, Silva LA. Riscos ocupacionais e alterações de saúde entre trabalhadores de enfermagem brasileiros de unidades de urgência e emergência. Cienc. enferm. 2010; XVI(2): 69-81.

5. Tarantola A, Abiteboul D, Rachline A. Infection risks following accidental exposu- re to blood or body fluids in health care workers: A review of pathogens transmitted in published cases. Am J Infect Control. 2006; 34(6): 367-75.

6. Lee R. Occupational transmission of bloodborne diseases to healthcare workers in developing countries: meeting the challenges. J Hosp Infect. 2009; 72(4): 285-91.

7. Almeida MCM, Canini SRMS, Reis RK, Toffano SEM, Pereira FMV, Gir E. Seguimento clínico de profissionais e estudantes da área da saúde expostos a material biológico potencialmente contaminado. Rev Esc Enferm USP. 2015; 49(2): 261-66.

8. Voide C, Darling KEA, Kenfak-Foguena A, Erard V, Cavassini M, Lazor-Blanchet C. Underreporting of needlestick and sharps injuries among healthcare workers in a Swiss University Hospital. Swiss Med Wkly. 2012; 42(13523): 1-7.

9. Ministério da Saúde (BR). Recomendações para terapia antirretroviral em adultos infectados pelo HIV- 2008. Suplemento III - Tratamento e prevenção 2008. Brasília (DF); 2010.

10. Ministério da Saúde (BR). Secretaria de Políticas de Saúde, Coordenação Nacional de DST e AIDS. Exposição a material biológico. Brasília (DF): Ministério da Saúde; 2006.

11. Martins AMEBL, Pereira RD, Ferreira RC. Adesão a protocolo pós exposição ocupacional de acidentes entre cirurgiões dentistas. Rev Saude Publica. 2010; 44(3): 528-540.

12. Araújo TME, Costa e Silva, N. Acidentes perfurocortantes e medidas preventivas para hepatite $\mathrm{B}$ adotadas por profissionais de Enfermagem nos serviços de urgência e emergência de Teresina, Piauí. Rev. bras. saúde ocup. 2014: 39(130): 175-83.

13. Oliveira AC, Diaz MEP, Toledo AD. Acidentes de trabalho com materiais perfurocortantes entre a equipe multiprofissional de uma unidade de emergência. Cienc Cuid Saude. 2010; 9(2): 341-49. 
14. Ministério da Saúde (BR). Conselho Nacional de Saúde. Resolução Nº 466, de 12 de dezembro de 2012. Aprova diretrizes e normas regulamentadoras de pesquisas envolvendo seres humanos. Brasília: Diário Oficial da União, Brasília, DF. 2013; 112(1677-9).

15. Machado MRM, Machado FM. Acidentes com material biológico em trabalhadores de enfermagem do Hospital Geral de Palmas (TO). Rev. bras. saúde ocup. 2011: 36(124): 274-81.

16. Toska A, Saridi M, Wozniak G, Rekleiti $\mathrm{M}$, Mouskou S, Souliotis K, et al. Incidence and frequency of mucocutaneous exposure and percutaneous injuries in Greek nurses: are they protected enough? Balkan Military Medical Review. 2014; 17(4): 120-25.

17. Neto JPS, Alexandre SMB, Sousa MNA. Acidentes de trabalho e subnotificações: estudo com enfermeiros atuantes na atenção terciária. Rev. Elet. Fainor. 2014; 7(2): 219-31.

18. Habib H, Khan EA, Aziz A. Prevalence and factors associated with needle stick injuries among registered nurses in public sector tertiary care hospitals of Pakistan. IJCRIMPH. 2011; 3(2): 124-30.

19. Voide C, Darling KEA, , Kenfak-Foguena A, Erard V, Cavassinia M, Lazor-Blanchet C. Underreporting of needlestick and sharps injuries among healthcare workers in a Swiss University Hospital. Swiss Med Wkly. 2012; 142: w13523.

20. Poveda VB, Guerra LS, Carvalho OLT, Silva PLM, Araújo MOP. Acidentes ocupacionais com profissionais da equipe de enfermagem de um hospital do vale do paraíba paulista. Rev Univers Vale Paraíba. 2011; 17(29): 118-32.

21. Pimenta FR, Ferreira MD, Gir E, Hayashida M, Canini SRMS. Atendimento e seguimento clínico especializado de profissionais de enfermagem acidentados com material biológico. Rev Esc Enferm USP. 2013; 47(1): 198-204.
22. Kessler CS, McGuinn M, Spec A, Christensen J, Baragi R, Hershow RC. Underreporting of blood and body fluid exposures among health care students and trainees in the acute care setting: A 2007 survey. Am J Infect Control. 2011; 39(2): 129-34.

23. Prochnow A, Magnago TSBS, Tavares JP, Beck CLC, Silva RM, Greco PBT. Perfil dos acidentes de trabalho publicados em estudos brasileiros. Saúde (Santa Maria). 2011; 37(1): 77-90.

24. Luize PB, Canini SRMS, Gir E, Toffano SEM. Condutas após exposição ocupacional a material biológico em um hospital especializado em oncologia. Texto contexto - enferm. 2015; 24(1): 170-77.

25. Sindhiya JT, Muhemmed A, Akhtar J, Shaikh J. M. Needle stick injuries among dental health care providers: a survey done at hyderabad and karachi. Pakistan Oral \& Dental Journal. 2014; 34(2): 33943.

26. Au E, Gossage JA, Bailey SR. The reporting of needlestick injuries sustained in theatre by surgeons: are we under-reporting? J Hosp Infect. 2008; 70(1): 66-70.

27. Santos AS, Araújo TME, Viana MRP, Santos MS , Araújo RRM, Campelo TPT. Needlestick injuries in nursing personnel of urgent and emergency services in a brazilian capital. Rev. pesq. cuid. fundam. (Online). 2011; (Ed.Supl.): 229-241.

28. Garbaccio JL, Regis WCB, Silva RMC, Estevão WG. Acidentes ocupacionais com a equipe de enfermagem da atenção hospitalar. Cogitare Enferm. 2015; 20(1): 14652.

29. Makary MA. et al. Needlestick Injuries among Surgeons in Training. NEJM. 2007; 356(26): 2693-699.

30. Azadi A, Anoosheh M, Delpisheh A. Frequency and barriers of underreported needlestick injuries amongst Iranian nurses, a questionnaire survey. J Clin Nurs. 2010; 20(3-4): 488-93. 